ARTICLE

https://doi.org/10.1038/s41467-019-12269-4

\title{
Organocatalytic atroposelective construction of axially chiral arylquinones
}

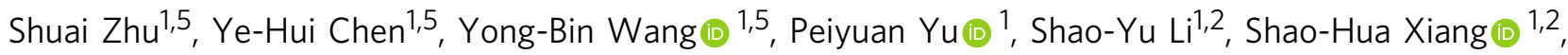 \\ Jun-Qi Wang (1) ${ }^{3}$, Jian Xiao (i) ${ }^{4} \&$ Bin Tan ${ }^{1}{ }^{1}$
}

\begin{abstract}
Atropisomeric biaryl motifs are ubiquitous in chiral catalysts and ligands. Numerous efficient strategies have been developed for the synthesis of axially chiral biaryls. In contrast, the asymmetric construction of o-quinone-aryl atropisomers has yet to be realized. Inspired by the rapid progress of the chemistry of biaryls, here we present our initial investigations about the atroposelective construction of axially chiral arylquinones by a bifunctional chiral phosphoric acid-catalyzed asymmetric conjugate addition and central-to-axial chirality conversion. With o-naphthoquinone as both the electrophile and the oxidant, three types of arylation counterparts, namely 2-naphthylamines, 2-naphthols and indoles, are utilized to assemble a series of atropisomeric scaffolds in good yields and excellent enantioselectivities. This approach not only expands the axially chiral library but also offers a route to a class of potential, chiral biomimetic catalysts.
\end{abstract}

\footnotetext{
${ }^{1}$ Shenzhen Grubbs Institute, Department of Chemistry, Southern University of Science and Technology, Shenzhen 518055, China. ${ }^{2}$ Academy for Advanced Interdisciplinary Studies, Southern University of Science and Technology, Shenzhen 518055, China. ${ }^{3}$ Department of Biology, Southern University of Science and Technology, Shenzhen 518055, China. ${ }^{4}$ College of Chemistry and Pharmaceutical Sciences, Qingdao Agricultural University, Qingdao 266109, China. ${ }^{5}$ These authors contributed equally: Shuai Zhu, Ye-Hui Chen, Yong-Bin Wang. Correspondence and requests for materials should be addressed to B.T. (email: tanb@sustc.edu.cn)
} 
E nantioenriched axially chiral biaryls are of great significance due to the ubiquitous relevance in natural products ${ }^{1,2}$, bioactive molecules ${ }^{3,4}$ and functional materials $s^{5,6}$. They also constitute the core structural motif of various chiral catalysts and ligands for asymmetric transformations ${ }^{7,8}$. Particularly, the emergence of BINOL $^{9,10}$ and its derivatives ${ }^{1-13}$ brought about remarkable improvement of asymmetric catalysis domain and altered the scenario that the acquirement of highly enantioenriched products from prochiral substrates via routinely chemical approaches remained extremely challenging half a century ago. The past couple of decades have witnessed the rapid progress of the biaryls based chemistry and a series of efficient synthetic strategies have been developed for the construction of chiral biaryls (Fig. 1a) ${ }^{14-16}$. In stark contrast, despite only slight alteration in molecular structure, the $o$-quinone-aryl atropisomer has been still unrevealed and its asymmetric synthesis has never been materialized till now, to the best of our knowledge. On the other hand, the $o$-quinones occur prevalently in bioactive natural products $^{17,18}$ and are widely recognized as cofactors in numerous quinoproteins (Fig. 1b) ${ }^{19-22}$. Mechanistic studies reveal the role of quinone cofactors as the actual active centers while metals necessitate the regeneration of the quinone cofactors in these transformations. In addition, they were found to be useful synthetic intermediates for many valuable transformations ${ }^{23,24}$. In this regard, the enantioselective construction of hitherto unknown atropisomeric aryl $o$-quinone not only brings about a class of axially chiral atropisomers but also may provide a class of potential chiral biomimetic catalysts.

On the basis of the recent advance in the construction of axially chiral backbones ${ }^{25-34}$ and our continuous understanding of this field ${ }^{35}$, we envisaged that the atroposelective synthesis of the axially chiral $o$-quinone is conceivable via arylation of $o$-naphthoquinone promoted by organocatalyst. First, enantioselective arylation of $o$-naphthoquinone I with aryl nucleophile II provides central chiral intermediate III in the presence of chiral organocatalyst. Subsequent aromatization and oxidation result in the transfer of central to axial chirality, furnishing the excepted axially chiral aryl $o$-naphthoquinone IV. Ideally, the oxidative capability of excess $o$-naphthoquinone I will facilitate the oxidation reaction (Fig. 1c). However, related research becomes more appealing and challenging when the planar aromatic ring was replaced by an $o$-quinone as compared to biaryls and then several challenges need to be taken into consideration: (1) appropriate substituents should be equipped on $o$-naphthoquinone and/or nucleophile to enhance the reactivity and restrict the axial rotation; (2) an appropriate bifunctional organocatalyst is essential for simultaneous activation of nucleophile and electrophile in appropriate chiral environment; strong background reaction is detrimental to the stereocontrol; (3) entire oxidation process should proceed under mild conditions to circumvent the racemization of the axial chirality.

Herein we disclose the discovery of axially chiral $o$-naphthoquinones and their enantioselective construction in the presence of bifunctional chiral phosphoric acid catalyst via central-to-axial chirality conversion. A range of atropisomeric $o$-naphthoquinone frameworks are synthesized in good yields and excellent enantiocontrol with $o$-naphthoquinone as the electrophile and oxidant, 2-naphthylamine, 2-naphthol or indole as the arylation reagent.

\section{Results}

Optimization of the reaction conditions. Considering the aforementioned factors, we selected $o$-naphthoquinone $\mathbf{1 a - 1}$ with an ester group as the model electrophile and 2-naphthylamine 2a-1 as the aryl donor to construct axially chiral backbone under the activation of chiral phosphoric acid ${ }^{36,37}$. The introduction of ester group was thought to enhance the interaction between quinone and catalyst while strengthens the stability of chiral axis of the product in line with the reported results ${ }^{38}$. Also, we expected the excessive $o$-naphthoquinone to serve as oxidant to de-aromatize naphthol intermediate. To verify the feasibility of our design, $10 \mathrm{~mol} \%$ of chiral phosphoric acid (CPA1) was utilized to promote the reaction of $\mathbf{1 a - 1}$ (2.2 eq.) and $\mathbf{2 a - 1}$ (1.0 eq.) in $\mathrm{CH}_{2} \mathrm{Cl}_{2}$ at room temperature. Fortunately, the desired product 3a-1 was afforded in $4 \mathrm{~h}$ in $32 \%$ isolated yield (Table 1, entry 1 ).
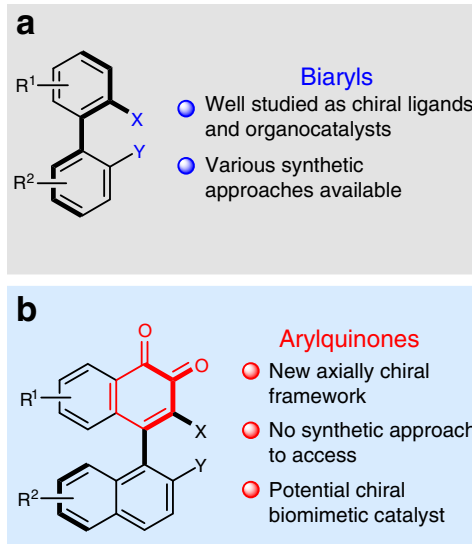

C

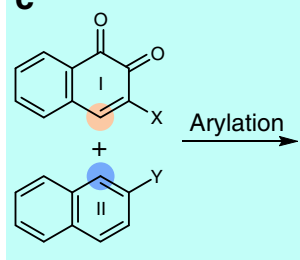

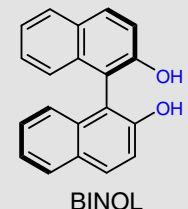

BINOL

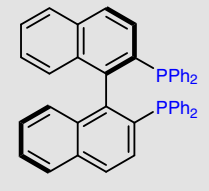

BINAP

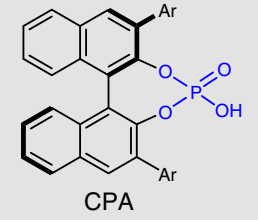

CPA
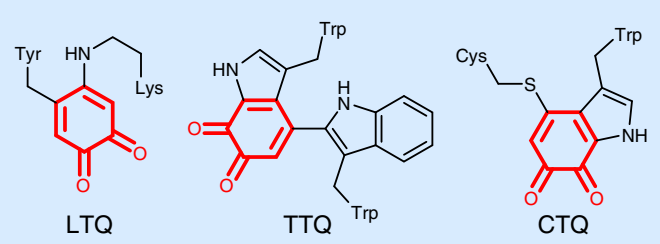

LTQ
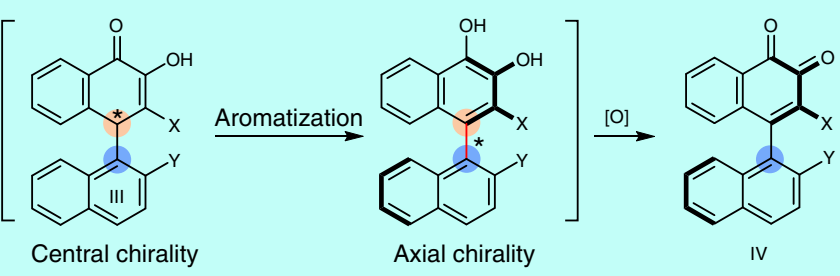

Fig. 1 Axially chiral biaryls vs. axially chiral arylquinones and their synthesis. a Biaryls as chiral ligands and organocatalysts. b o-Quinone structures in biomimetic catalysts. c Our designed approach to access arylquinone atropisomer 


\section{Table 1 Reaction optimization with 2-naphthylamine ${ }^{a}$}

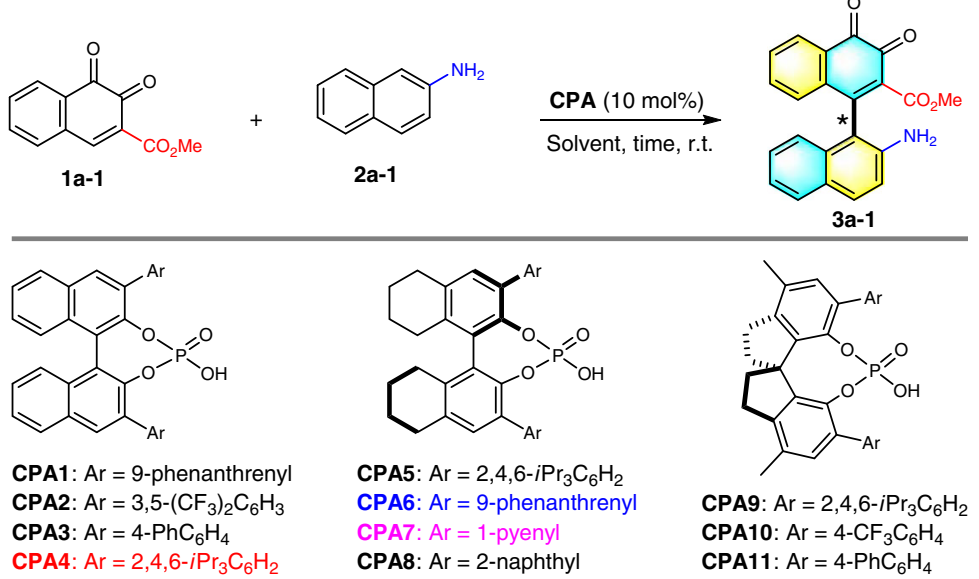

\begin{tabular}{|c|c|c|c|c|c|}
\hline entry & CPA & solvent & time (min) & yield $(\%)^{b}$ & ee $(\%)^{c}$ \\
\hline 1 & $(R)$-CPA1 & $\mathrm{CH}_{2} \mathrm{Cl}_{2}$ & 240 & 32 & -4 \\
\hline 2 & $(R)-\mathbf{C P A 2}$ & $\mathrm{CH}_{2} \mathrm{Cl}_{2}$ & 240 & 38 & -36 \\
\hline 3 & $(R)-\mathbf{C P A 3}$ & $\mathrm{CH}_{2} \mathrm{Cl}_{2}$ & 240 & 27 & -51 \\
\hline 4 & (S)-CPA4 & $\mathrm{CH}_{2} \mathrm{Cl}_{2}$ & 20 & 87 & 99 \\
\hline 5 & $(R)-\mathbf{C P A 5}$ & $\mathrm{CH}_{2} \mathrm{Cl}_{2}$ & 20 & 85 & 99 \\
\hline 6 & (R)-CPA6 & $\mathrm{CH}_{2} \mathrm{Cl}_{2}$ & 240 & 36 & -83 \\
\hline 7 & (R)-CPA7 & $\mathrm{CH}_{2} \mathrm{Cl}_{2}$ & 240 & 57 & -47 \\
\hline 8 & $(R)-\mathbf{C P A} \mathbf{8}$ & $\mathrm{CH}_{2} \mathrm{Cl}_{2}$ & 240 & 52 & -30 \\
\hline 9 & (R)-CPA9 & $\mathrm{CH}_{2} \mathrm{Cl}_{2}$ & 240 & 24 & -28 \\
\hline 10 & $(R)-\mathbf{C P A 1 0}$ & $\mathrm{CH}_{2} \mathrm{Cl}_{2}$ & 240 & 35 & -14 \\
\hline 11 & (R)-CPA11 & $\mathrm{CH}_{2} \mathrm{Cl}_{2}$ & 240 & 38 & -42 \\
\hline 12 & (S)-CPA4 & toluene & 20 & 60 & 99 \\
\hline 13 & (S)-CPA4 & $\mathrm{CHCl}_{3}$ & 20 & 82 & 99 \\
\hline 14 & (S)-CPA4 & EtOAc & 20 & 43 & 68 \\
\hline 15 & (S)-CPA4 & DCE & 20 & 86 & 99 \\
\hline 16 & (S)-CPA4 & ether & 20 & 62 & 83 \\
\hline $17^{d}$ & (S)-CPA4 & $\mathrm{CH}_{2} \mathrm{Cl}_{2}$ & 20 & 86 & 99 \\
\hline $18^{e}$ & (S)-CPA4 & $\mathrm{CH}_{2} \mathrm{Cl}_{2}$ & 20 & 86 & 99 \\
\hline
\end{tabular}

a Reaction conditions: 1a-1 $(0.22 \mathrm{mmol}), \mathbf{2 a - 1}(0.10 \mathrm{mmol})$, solvent $(2 \mathrm{~mL})$ under Ar atmosphere, unless noted otherwise. ${ }^{b}$ Isolated yields. ${ }^{\mathrm{C}} \mathrm{Determined}$ by $\mathrm{HPLC}$ analysis on a chiral stationary phase. ${ }^{d}$ Conducted with $5 \mathrm{~mol} \%$ of CPA4. ${ }^{e}$ Conducted with $1 \mathrm{~mol} \%$ of CPA4

Notably, this compound displayed axial chirality based on the chiral stationary high-performance liquid chromatography (HPLC) analysis. Despite only inapparent enantiomeric excess ( $4 \%$ ee), our proposed hypothesis was substantiated by direct experimental evidence and the enantioenriched atropisomeric $o$ naphthoquinone was realized. Following optimization focused on the evaluation of various CPA and BINOL-derived CPA4 with a bulky 2,4,6-triisopropylphenyl substituent on the 3,3'-position was found to be the best catalyst, providing 3a-1 in excellent yield and enantioselectivity ( $87 \%$ yield, $99 \%$ ee) in only $20 \mathrm{~min}$ (Table 1, entries 2-11). Other solvents were surveyed to further improve the reaction but in futile (Table 1, entries 12-16). Further investigations revealed that $1 \mathrm{~mol} \%$ catalyst loading was optimal to uphold the high yield, as well as enantiocontrol of the current reaction (Table 1, entry 18). The optimized conditions were then concluded as follows: 1a-1 $(0.22 \mathrm{~mol}), \mathbf{2 a}-\mathbf{1}(0.10 \mathrm{~mol})$ and CPA4 (1 mol\%) in $2 \mathrm{~mL}$ of $\mathrm{CH}_{2} \mathrm{Cl}_{2}$ and performed at room temperature for $20 \mathrm{~min}$.

Substrate scope. With the optimized conditions in hand, we next set out to explore the substrate scope of this reaction by examining derivatives of 2-naphthylamine and $o$-naphthoquinone. Overall, all the tested substrates gave the expected axially chiral arylquinones in good yields with excellent atroposelectivities
(Table 2). Firstly, replacement of methyl group on ester by an ethyl or benzyl group provided the adduct $\mathbf{3 a}-\mathbf{2}$ or $\mathbf{3 a}-\mathbf{3}$ in lower yield and enantioselectivity. The introduction of a substituent on the aromatic ring of $o$-naphthoquinones (3a-4 3a-7) led to slight yield reduction. A wide range of 2-naphthylamines with different substituents or substitution patterns were evaluated under the standard conditions. In most cases, the desired axially chiral arylquinones $(\mathbf{3 a - 8} \mathbf{3} \mathbf{a}-\mathbf{1 4})$ could be furnished with up to $99 \%$ ee. A certain degree of variation in chemical yield was detected for each reaction. Notably, no desired product was obtained under standard conditions when $\mathrm{N}, \mathrm{N}$-dimethyl-2naphthylamine was used, indicating the importance of $\mathrm{N}-\mathrm{H}$ bond for this reaction.

To further expand the generality of this strategy, we applied the optimized conditions to the reaction of $o$-naphthoquinone $\mathbf{1 a - 1}$ and phenyl protected 2-naphthylamine $\mathbf{2 b}-\mathbf{1}$. Disappointedly, less than $10 \%$ of conversion was detected after $10 \mathrm{~h}$ under this set of conditions. While previous studies suggested the indicative effects of CPA catalyst on reaction outcomes, the re-optimization was initiated by screening of various CPA. Our optimization efforts revealed that $\mathrm{H}_{8}-\mathrm{BINOL}$ derived CPA6 could provide the most optimal results at room temperature for $10 \mathrm{~h}$, affording the product $\mathbf{3 b}-\mathbf{1}$ in $93 \%$ yield and $99 \%$ ee. Further studies showed $\mathrm{CH}_{2} \mathrm{Cl}_{2}$ remained as the most efficient reaction solvent. While 10 mol\% catalyst loading could not bring about any improvement 
Table 2 Substrate scope with 2-naphthylamine ${ }^{a}$

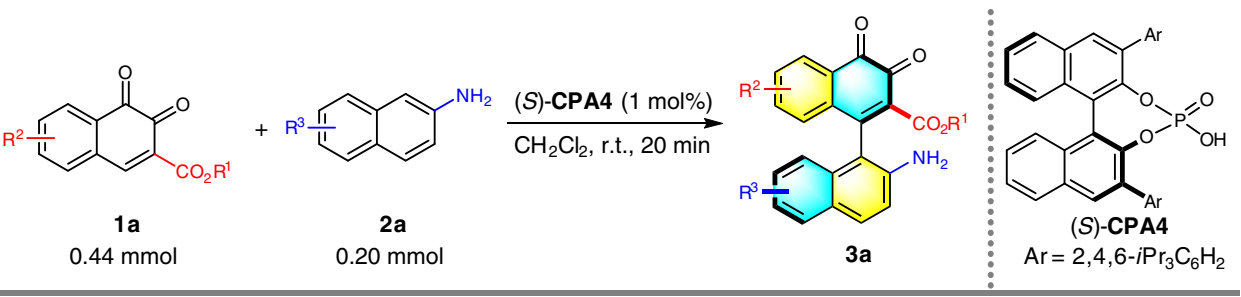

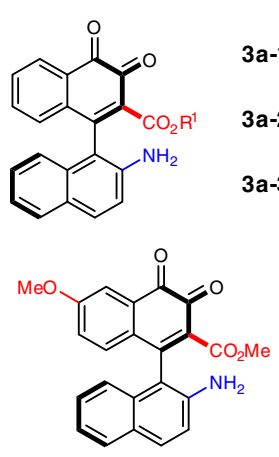

3a-7, $70 \%$ yield, $96 \%$ ee

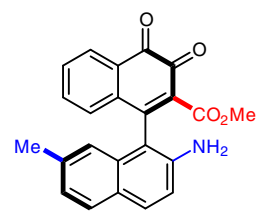

3a-11, $72 \%$ yield, $99 \%$ ee
$3 a-1, R^{1}=M e, 86 \%$ yield, $99 \%$ ee

a-2, $R^{1}=E t, 83 \%$ yield, $97 \%$ ee

a-3, $R^{1}=$ Bn, $82 \%$ yield, $93 \%$ ee

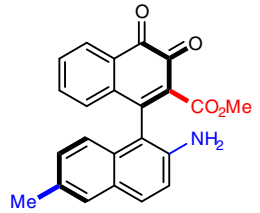

$3 a-8,73 \%$ yield, $98 \%$ ee

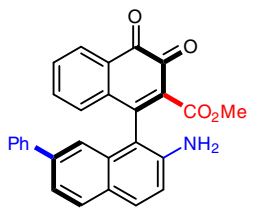

3a-12, 75\% yield, $99 \%$ ee

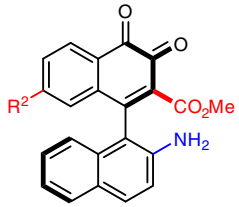

$3 \mathrm{a}-4, \mathrm{R}^{2}=n \mathrm{Bu}, 76 \%$ yield, $98 \%$ ee

3a-5, $\mathrm{R}^{2}=\mathrm{Ph}, 80 \%$ yield, $99 \%$ ee

3a-6, $R^{2}=B r, 80 \%$ yield, $96 \%$ ee<smiles>COC(=O)C1=C(c2c(N)ccc3cc(OC)ccc23)c2ccccc2C(=O)C1=O</smiles>

$3 a-9,75 \%$ yield, $99 \%$ ee

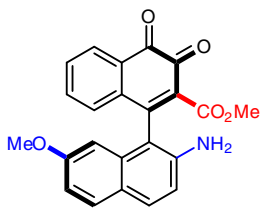

3a-13, $81 \%$ yield, $99 \%$ ee

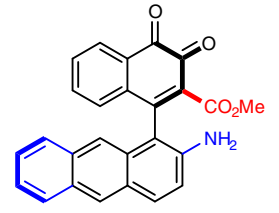

3a-10, $87 \%$ yield, $95 \%$ ee

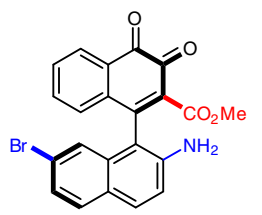

$3 a-14,72 \%$ yield, $99 \%$ ee

a Reaction conditions: $\mathbf{1 a}(0.44 \mathrm{mmol}), \mathbf{2 a}(0.20 \mathrm{mmol}),(\mathrm{S})$-CPA4 $(1 \mathrm{~mol} \%), \mathrm{CH}_{2} \mathrm{Cl}_{2}(4 \mathrm{~mL})$, room temperature for 20 min under Ar atmosphere. Isolated yields were provided and ee values were determined by HPLC analysis on a chiral stationary phase

for the reaction results, $1 \mathrm{~mol} \%$ catalyst loading resulted in chemical yield decrement of $3 \mathrm{~mol} \%$ (Supplementary Table 2). The re-optimized conditions were then applied to a wide spectrum of $o$-naphthoquinones and phenyl protected 2naphthylamines (Table 3). The influence of the ester groups on the $o$-naphthoquinone was first investigated. Slightly compromised reaction yield and selectivity were observed when methyl group was replaced with bulkier ethyl (3b-2), benzyl (3b-3) or isopropyl group (3b-4). Nonetheless, additional substituent introduced on the aromatic ring of $o$-naphthoquinone exerted limited effects to the reaction outcomes (3b-5 3b-8). Halide functionality (3b-9 to 3b-11), electron-donating (3b-12 and $\mathbf{3 b}-\mathbf{1 3})$ and electron-withdrawing groups (3b-14) on phenyl protecting group were all well tolerated to give the corresponding products in satisfactory results. To verify the utility of the current organocatalytic atroposelective reaction, a gram-scale synthesis of 3b-7 was performed under the standard reaction conditions. As presented in Table 3, the desired axially chiral product was furnished with the same enantiocontrol but in higher yield, inferring this protocol is suitable for the large-scale production.

In our original design, the ester group was proposed to enhance the interaction between CPA and substrate as well as the stability of the chiral axis. However, the C-3 substituent of o-naphthoquinones has been demonstrated to reduce the catalytic capability significantly ${ }^{39}$. Encouraged by our initial results, we then attempted to explore the practicability for the synthesis of axially chiral arylquinones without an ester group. The more challenging and rewarding project commenced with $o$-naphthoquinone $\mathbf{1 b}-\mathbf{1}$ as the model electrophile. Compound $\mathbf{2 b - 1}$ was anticipated to be an appropriate nucleophile since the bulky protecting group might be conducive to restrict the axial rotation. The reaction was conducted in $\mathrm{CH}_{2} \mathrm{Cl}_{2}$ with $10 \mathrm{~mol} \% \mathbf{C P A} 4$ as the catalyst. Gratifyingly, the desired product 3c-1 was provided in $92 \%$ yield and $83 \%$ ee. No improved result arose by screening a variety of CPAs including CPA6 (78\% yield, $46 \%$ ee), the optimal catalyst for the reaction of phenyl protected 2-naphthylamines and $o$ naphthoquinones with an ester group. Not surprisingly, $\mathrm{CH}_{2} \mathrm{Cl}_{2}$ proved to be the most efficient solvent. The enantioselectivity was augmented to $93 \%$ when the reaction was performed at $-30{ }^{\circ} \mathrm{C}$, albeit lower yield (63\%). The reduction of the catalyst loading led to obvious yield and enantioselectivity depletion (Supplementary Table 3). The optimal reaction conditions were then applied to other four substrates. As depicted in Table 4, all these reactions could deliver the desired products $(3 c-2$ to $3 c-5)$ in excellent stereocontrol at the expense of lower yields. Although limited substrate scope was established at this stage, the successful construction of this axially chiral backbone offered more possibility to realize the application in catalysis.

To further broaden the scope of axially chiral arylquinone family, we moved to seek other applicable nucleophiles upon accomplishing the investigation with 2-naphthylamine and its derivatives. 2-Naphthol, which has proven impressive ability to act as aryl nucleophiles for construction of BINOL derivatives $^{40,41}$, then came to our mind. Employing 2-naphthol $2 \mathrm{c}$ as the aryl donor, we again envisaged the prohibitory role of an ester moiety on $o$-naphthoquinone for hindering the rotation of chiral axis. Careful screening of the conditions presented the following optimal system: $\mathrm{H}_{8}-\mathrm{BINOL}$-derived CPA7 as the catalyst, $\mathrm{BF}_{4} \mathrm{Li}$ as the additive, and the reaction was conducted in $\mathrm{CH}_{2} \mathrm{Cl}_{2}$ at $-10^{\circ} \mathrm{C}$ for $24 \mathrm{~h}$ to give the desired product $\mathbf{3 d}-\mathbf{1}$ in 


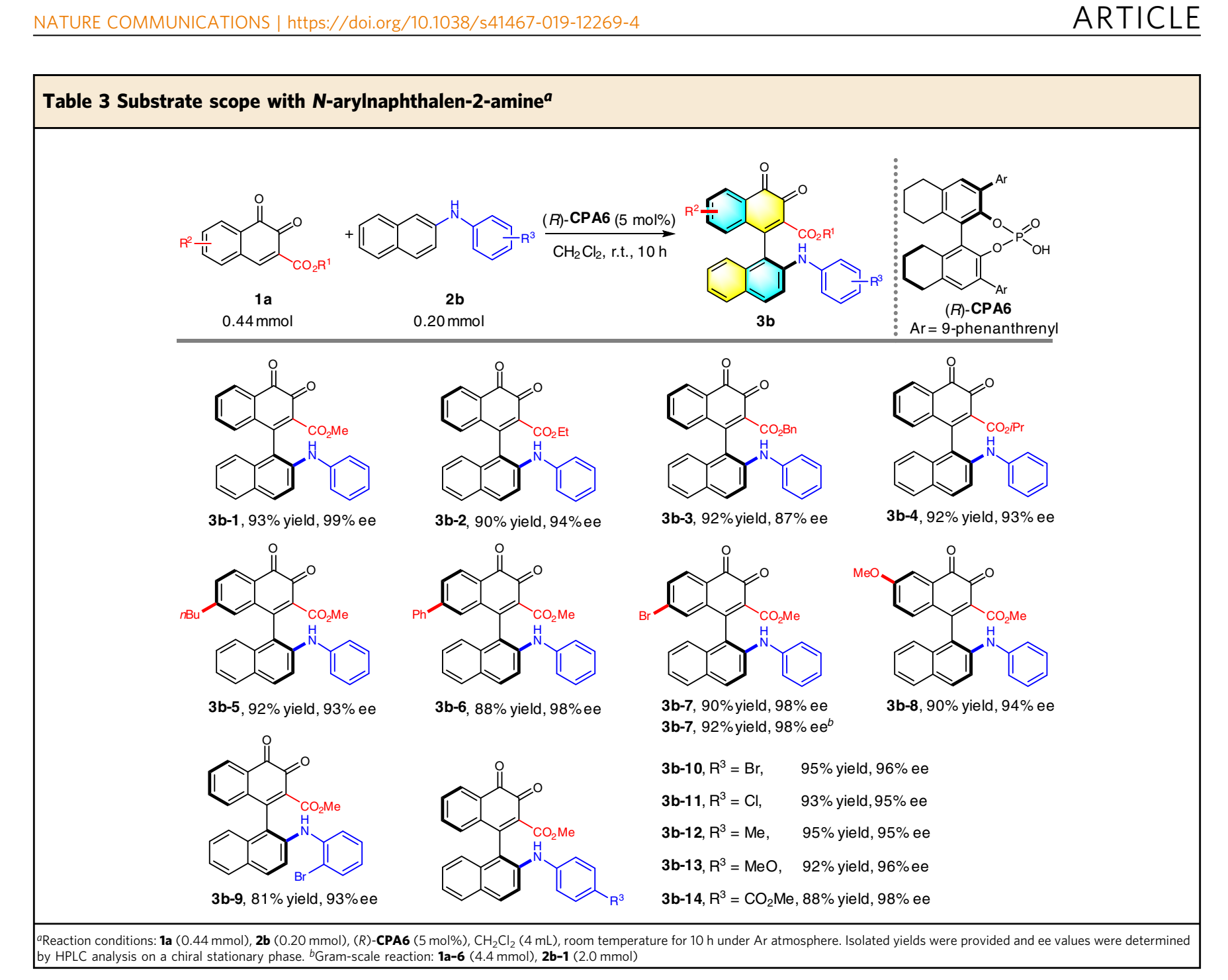

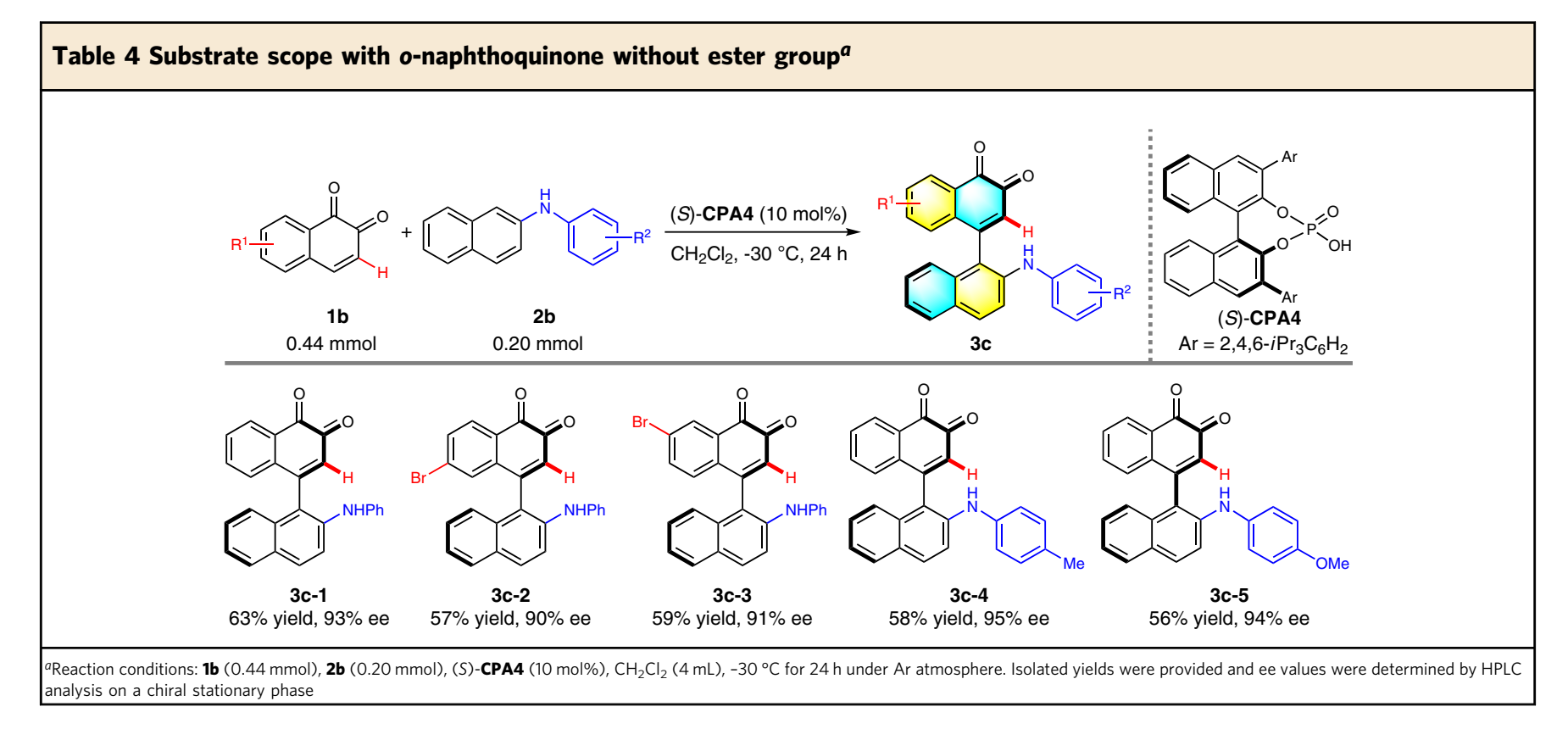


Table 5 Substrate scope with 2-naphthola

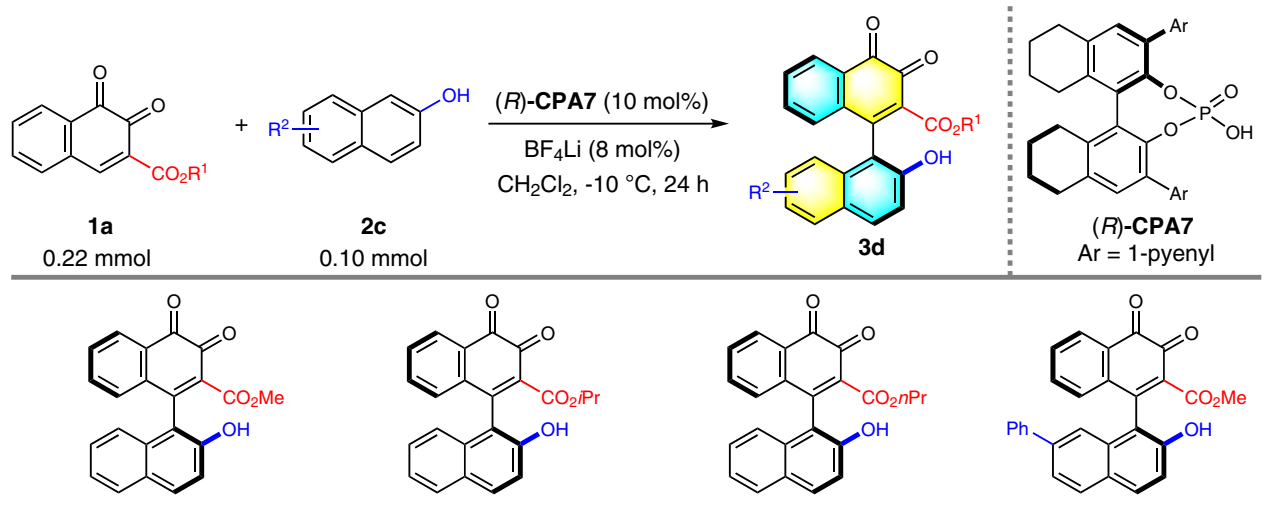

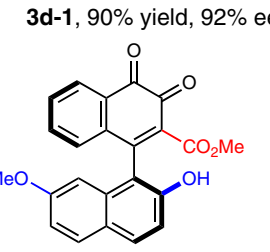

3d-5, $90 \%$ yield, $89 \%$ ee 3d-2, 93\% yield, $94 \%$ ee

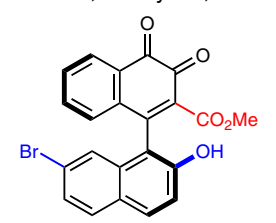

3d-6, $92 \%$ yield, $89 \%$ ee 3d-3, 91\% yield, 93\% ee

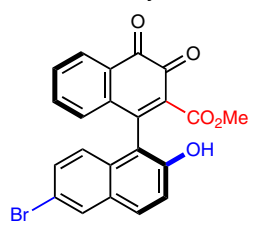

3d-7, 91\% yield, $90 \%$ ee

aReaction conditions: $\mathbf{1 a}(0.22 \mathrm{mmol}), \mathbf{2 c}(0.10 \mathrm{mmol}),(R)-\mathbf{C P A 7}(10 \mathrm{~mol} \%), \mathrm{CH}_{2} \mathrm{Cl}_{2}(4 \mathrm{~mL}), \mathrm{BF}_{4} \mathrm{Li}(8 \mathrm{~mol} \%),-10{ }^{\circ} \mathrm{C}$ for $24 \mathrm{~h}$ under $\mathrm{Ar}$ atmosphere. Isolated yields were provided and ee values were determined by HPLC analysis on a chiral stationary phase

90\% yield and $92 \%$ ee (Supplementary Table 5). This protocol has shown excellent applicability and compatibility to several analogs (Table 5). All products (3d-2 3d-7) were obtained in excellent yields and enantioselectivities respectively.

Besides, indole has been validated to be a competent nucleophile ${ }^{42,43}$ under acidic conditions for the synthesis of indolylbenzoquinone $e^{44,45}$, which is widespread in various natural products with remarkable bioactivity. However, to the best of our knowledge, there is no facile asymmetric construction of axially chiral indolyl-o-naphthoquinone to-date. To test the relevance of indole nucleophile in our protocol, CPA4 $(10 \mathrm{~mol} \%)$ was utilized to promote the reaction of $\mathbf{1 a - 1}$ and $\mathbf{2 d - 1}$ in $\mathrm{CH}_{2} \mathrm{Cl}_{2}$ for $24 \mathrm{~h}$ under Ar atmosphere which led to the corresponding product in $44 \%$ ee. Extensive efforts dedicated in a massive parallel screening of conditions were unfruitful with only moderate enantiocontrol (76\% ee) attained. Interestingly, the bromide-containing substrate 1c-1 provided the product $\mathbf{3 e - 1}$ efficiently with $89 \%$ ee with the optimal catalyst CPA4 in $\mathrm{CH}_{2} \mathrm{Cl}_{2}$. Subsequent optimization further affirmed the superior reaction competency of $\mathrm{CH}_{2} \mathrm{Cl}_{2}$ compared to other solvents in the presence of CPA4. The reaction selectivity was dictated by catalyst loading in which lower catalyst loading gave lower reaction efficiency in return. Notably, the enantioselectivity was improved to $96 \%$ ee when the reaction was performed at $0{ }^{\circ} \mathrm{C}$ without diminution of the yield (95\%). On the contrary, alteration of other factors such as reaction time and molarity proved futile. The optimized conditions were then concluded as follows: 1c-1 $0.20 \mathrm{~mol}, \mathbf{2 d}-\mathbf{1} 0.10 \mathrm{~mol}, 10 \mathrm{~mol} \%$ CPA4 in $\mathrm{CH}_{2} \mathrm{Cl}_{2}$ and the reaction was performed at $0{ }^{\circ} \mathrm{C}$ for $24 \mathrm{~h}$ under air atmosphere (Supplementary Table 6).

With the optimal conditions in hand, we next explored the substrate scope and the limitation of this reaction. Firstly, the effect of the substituent on the indole ring was tested. As summarized in Table 6, indole nucleophile with either an electron donating group $(\mathbf{3 e}-\mathbf{2}, \mathbf{3 e - 3})$ or a halide group $(\mathbf{3 e}-\mathbf{4}, \mathbf{3 e}-\mathbf{5})$ could be utilized to give the desired product in excellent yields and enantioselectivities. Likewise, both chlorine (3e-6) and iodine group (3e-7) substituted $o$-naphthoquinone electrophiles were very well tolerated under the conditions to give the axially chiral products with similar outcome and high chemical stability apart from the bromine counterpart. The isopentyl group could also act as the axial rotation resistance group to furnish product $\mathbf{3 e - 8}$ in consistently excellent enantioselectivity. Noteworthy, the stereoselectivity was completely lost $(0 \%$ ee $)$ when 2-tert-butyl-1methylindole $\mathbf{2 d - 7}$ was used as the nucleophile for this reaction, indicating that the $\mathrm{N}-\mathrm{H}$ bond of indole is crucial on the stereocontrol.

Transformations and asymmetric catalysis applications. To demonstrate the potential of the developed approach, synthetic transformations were then performed based on the generated products $\mathbf{3 b}$. Phenazine scaffold constitutes the core structures of various natural products with attractive bioactivities ${ }^{46-48}$. Moreover, it could sever as essential backbone for synthetic dyes. Accordingly, the synthesis of phenazine containing molecules to enrich the diversity of the library is of significant importance. Gratifyingly, the expected phenazine could be readily attained from $o$-quinone. The reactions of representative $\mathbf{3 b}$ with benzene1,2-diamine in the presence of concentrated $\mathrm{HCl}$ and sodium sulfate proceeded smoothly to yield the corresponding products $\mathbf{4 b}$ in quantitative yields with almost identical enantioselectivities (Fig. 2a). Subsequently, sodium dithionite was utilized to reduce the $o$-quinone moiety. The generated $o$-dihydroxybenzene intermediates were then trapped by $\operatorname{Tf}_{2} \mathrm{O}$ to give the more stable structures $\mathbf{5 b}$ in quantitative yields. Negligible deteriorations of stereochemical integrity were detected for all these reactions (Fig. 2a). Notably, the resultant phenyl trifluoromethanesulfonate provides opportunities for downstream coupling reactions, allowing quick access to a variety of structurally diversified atropisomeric binaphthyls. Interestingly, utilizing $\mathbf{5 b - 1}$ as the starting material, the Sonogashira coupling reaction proceeded smoothly to give bis-substituted product $\mathbf{6}$ with ethynyltrimethylsilane in $92 \%$ yield, while axially chiral molecule 7 with 


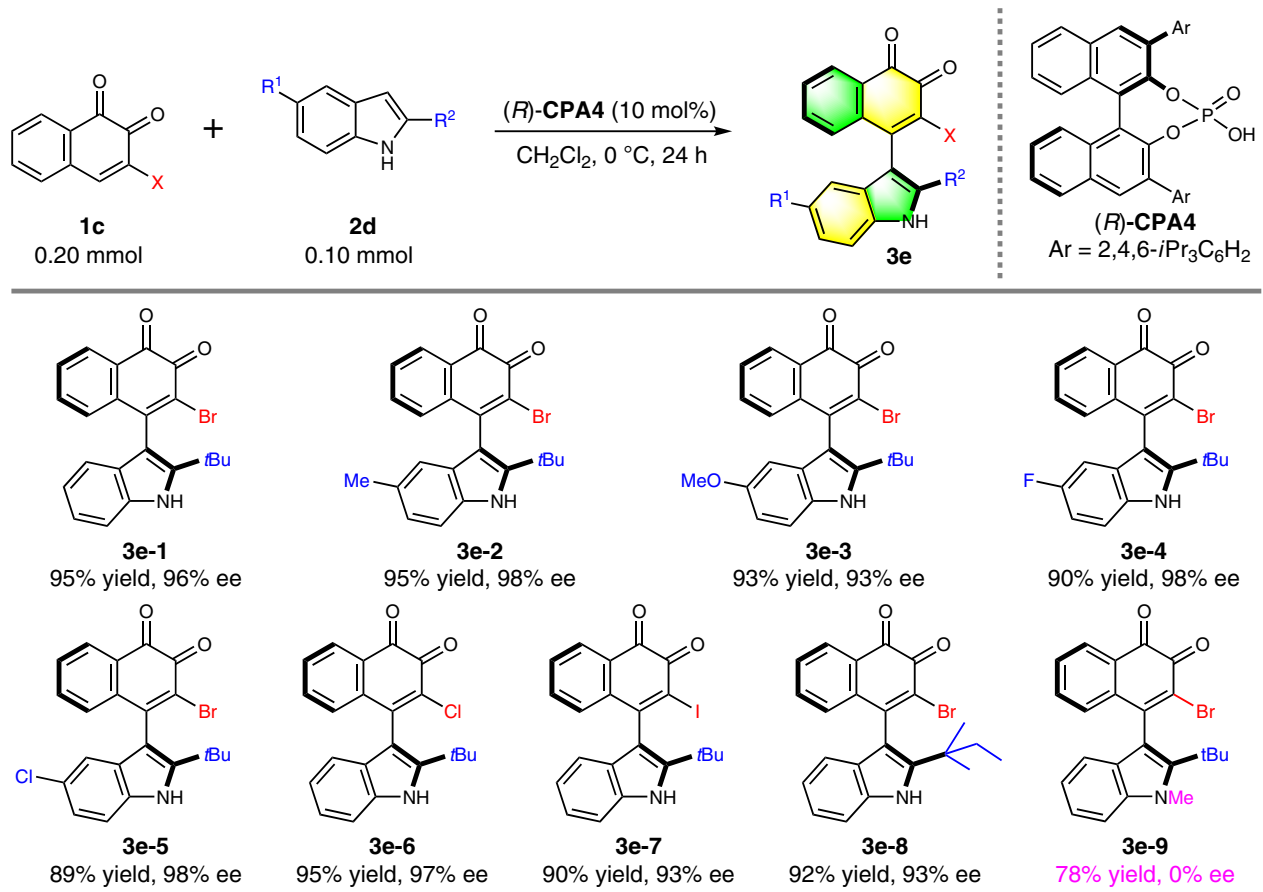

Reaction conditions: $\mathbf{1 c}(0.20 \mathrm{mmol}), \mathbf{2 d}(0.10 \mathrm{mmol}),(R)$-CPA4 $(10 \mathrm{~mol} \%)$ in $\mathrm{CH}_{2} \mathrm{Cl}_{2}(1 \mathrm{~mL})$ at $0{ }^{\circ} \mathrm{C}$ for $24 \mathrm{~h}$ under Ar atmosphere. Isolated yields were provided and ee values were determined by $\mathrm{HPLC}$ analysis on a chiral stationary phase

one olefinic group was produced efficiently by the Heck reaction. Apart from that, the Suzuki coupling reaction provided a mixture of $\mathbf{8 a}$ and $\mathbf{8 b}$ with $n$-butylboronic acid as the coupling partner. Likewise, no stereochemical integrity loss was observed for all these reactions (Fig. 2b). Next, the absolute configuration of $\mathbf{3 b - 1}$ was deduced to be $(S)$ by X-ray crystallographic analysis of its derivative $\mathbf{9}$, which could be prepared by the treatment of $\mathbf{3 b - 1}$ with (1 R,2 R)-1,2-diphenylethane-1,2-diamine under the identical conditions for the synthesis of compounds 4 (CCDC: 1833698), and those of other products (3a-3e) were assigned by analogy in accordance with the corresponding absolute configuration of CPAs. Subsequently, the ester group could be readily converted to alcohol with lithium aluminum hydride as the reductive agent, affording arylquinone $\mathbf{1 0}$ or $\mathbf{1 1}$ with acceptable yield, respectively (Fig. 2c). Finally, the synthetic and catalytic applications of the obtained axially chiral $o$-naphthoquinones were investigated. As shown in Fig. 2d, compound 12, prepared from compound $\mathbf{1 0}$ by reductive amination, acted as an oxidant for the transformation of benzylamine $\mathbf{1 3}$ to corresponding aldehyde. Subsequently, it severed as a chiral ligand in the presence of reducing agent for the asymmetric addition of $\mathrm{ZnEt}_{2}$ to the generated aldehyde, give the desired chiral alcohol 14 in moderate yield for two steps, albeit low enantioselectivity.

Plausible mechanism. Based on the experimental results and the elegant work of Rodriguez and Bonne involving central-to-axial chirality conversion strategy 49,50 , a plausible reaction pathway was proposed in Fig. 3. The first step of this reaction is the chiral phosphoric acid catalyzed asymmetric conjugate addition to form intermediate I with two chiral centers. The following rearomatization with central-to-axial chirality conversion gives atropisomeric intermediate II. Finally, oxidation by the use of excessive $o$-quinone furnished the excepted axially chiral arylquinone. Chiral phosphoric acid performed as a bifunctional organocatalyst to simultaneously activate 2-naphthylamine/2naphthol/indole and $o$-naphthoquinone by multiple $\mathrm{H}$-bonding and promote the first step of enantioselective nucleophilic addition to produce intermediate I. The rapid re-aromatization of this intermediate leads to the axially chiral intermediate II and eventually to the final product.

\section{Discussion}

In summary, we have discovered the axial chirality of $o$-naphthoquinones and provided the enantioselective conjugate addition strategy for the construction of axially chiral arylquinones with chiral phosphoric acid as the catalyst via sequential conjugated addition, chirality transfer and oxidation. With $o$-naphthoquinone as the electrophile and oxidant, three types of arylation nucleophiles, 2-naphthylamines, 2-naphthols, as well as indoles, were efficiently employed to outline the spectrum for axially chiral skeletons in good yields and excellent enantioselectivities. The resulted products could be converted to phenazine containing molecules and other useful axial chiral binaphthyls by downstream transformations. The arylquinone is not only an efficient reagent for the oxidation of amine, but also an applicable chiral ligand in asymmetric catalysis. Further investigations are underway to exploit the catalytic and synthetic applications in our laboratory.

\section{Methods}

General procedure for asymmetric synthesis $(\boldsymbol{R})$-3a. To a mixture of $(S)$-CPA4 (1 mol\%), $o$-naphthoquinone $1 \mathbf{a}(0.44 \mathrm{mmol})$ and nucleophile $\mathbf{2 a}(0.20 \mathrm{mmol})$ in $10 \mathrm{~mL}$ Schlenk tube was added $\mathrm{CH}_{2} \mathrm{Cl}_{2}(4.0 \mathrm{~mL})$ under Ar atmosphere. Then the reaction mixture was stirred vigorously at room temperature until 2 a was completely consumed. Typical reaction time was about $20 \mathrm{~min}$. The resulting mixture was concentrated under reduced pressure and purified by flash chromatography on silica gel (eluent: $\mathrm{PE} / \mathrm{EA}=4 / 1$ ) to afford the corresponding axially chiral arylquinones $(R)-\mathbf{3 a}$ 


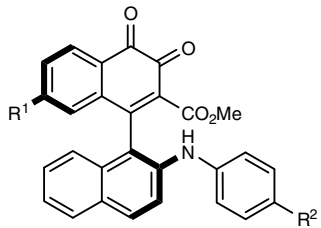

3b-1, $R^{1}=H, R^{2}=H, 99 \%$ ee 3b-7, $R^{1}=B r, R^{2}=H, 98 \%$ ee 3b-12, $R^{1}=H, R^{2}=M e, 95 \%$ ee $3 b-14, R^{1}=H, R^{2}=\mathrm{CO}_{2} \mathrm{Me}, 98 \%$ ee<smiles>CC(=O)c1cc2cc([Tl])ccc2c2nc3ccccc3nc12</smiles><smiles>CCCCc1c(Nc2ccc(Br)cc2)ccc2ccccc12</smiles>

OTf<smiles>O=C(O)c1c(O)c(O)c2ccc(Br)cc2c1-c1c(Nc2ccc(I)cc2)ccc2ccccc12</smiles>

$4 \mathbf{b}-1,99 \%$ yield, $94 \%$ ee $4 b-7,99 \%$ yield, $96 \%$ ee $4 b-12,99 \%$ yield, $96 \%$ ee 4b-14, $99 \%$ yield, $98 \%$ ee

\section{b}

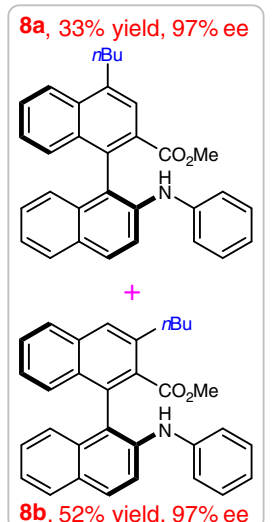

(1) aq. $\mathrm{Na}_{2} \mathrm{~S}_{2} \mathrm{O}_{4}, \mathrm{DCM}, 0^{\circ} \mathrm{C}$ (2) Pyridine, $\mathrm{Tf}_{2} \mathrm{O}, \mathrm{DCM},-78^{\circ} \mathrm{C}$

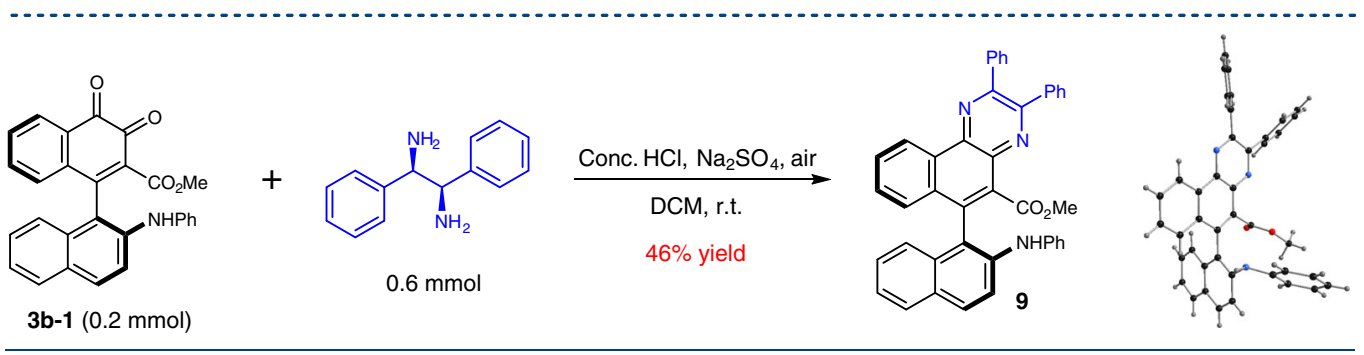

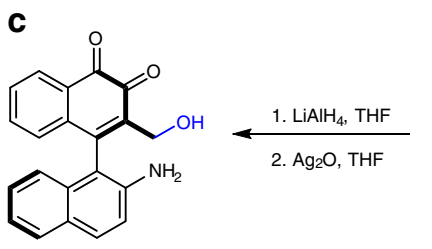

$10,60 \%$ yield, $99 \%$ ee

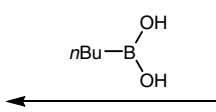

$\mathrm{Pd}\left(\mathrm{PPh}_{3}\right)_{4}$, DIPEA dioxane, $80^{\circ} \mathrm{C}, 6 \mathrm{~h}$
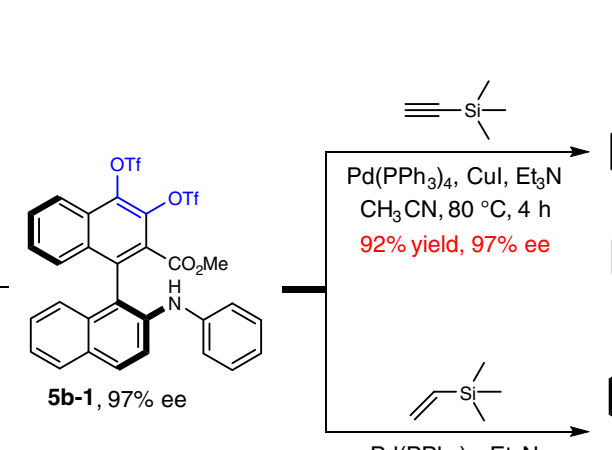

$\mathrm{Pd}\left(\mathrm{PPh}_{3}\right)_{4}, \mathrm{Et}_{3} \mathrm{~N}$ dioxane, $80^{\circ} \mathrm{C}, 4 \mathrm{~h}$ $80 \%$ yield, $97 \%$ ee<smiles>CC(C)(C)C#Cc1c(C(=O)O)c(-c2c(Nc3ccccc3)ccc3ccccc23)c2ccccc2c1C#CC(C)(C)C</smiles>

d

(2) $12(7 \mathrm{~mol} \%), \mathrm{Et}_{2} \mathrm{Zn}$, toluene, $28^{\circ} \mathrm{C}$

13

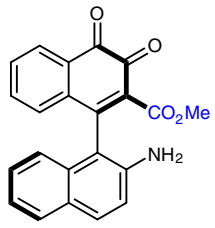

3a-1, 99\% ee<smiles>CC(=O)C1=C(c2c(Nc3ccccc3)ccc3ccccc23)c2ccccc2C(=O)C1=O</smiles>

3b-1, 99\% ee

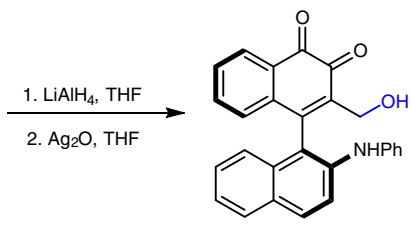

$11,67 \%$ yield, $96 \%$ ee

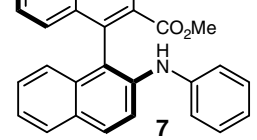




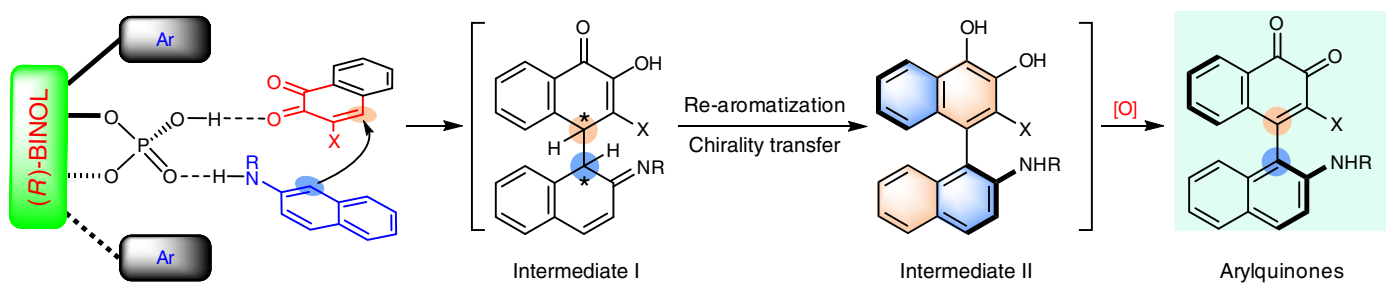

Fig. 3 Proposed reaction pathway. The reaction sequence follows chiral phosphoric acid catalyzed asymmetric conjugate addition, re-aromatization with central-to-axial chirality conversion and oxidation

General procedure for asymmetric synthesis of $(\boldsymbol{S})-\mathbf{3 b}$. To a mixture of $(R)$ CPA6 (5 mol\%), $o$-naphthoquinone $\mathbf{1 a}(0.44 \mathrm{mmol})$ and nucleophile $\mathbf{2 b}(0.20 \mathrm{mmol})$ in $10 \mathrm{~mL}$ Schlenk tube was added $\mathrm{CH}_{2} \mathrm{Cl}_{2}(4.0 \mathrm{~mL})$ under $\mathrm{Ar}$ atmosphere. Then the reaction mixture was stirred vigorously at room temperature until $\mathbf{2 b}$ was completely consumed. Typical reaction time was about $10 \mathrm{~h}$. The resulting mixture was concentrated under reduced pressure and purified by flash chromatography on silica gel (eluent: $\mathrm{PE} / \mathrm{EA}=6 / 1$ ) to yield the corresponding axially chiral arylquinones $(S)-\mathbf{3 b}$.

General procedure for asymmetric synthesis of $(\boldsymbol{R})$-3c. To a mixture of $(S)$ CPA4 $(10 \mathrm{~mol} \%)$ and nucleophile $2 \mathbf{b}(0.20 \mathrm{mmol})$ in $10 \mathrm{~mL}$ Schlenk tube was added $\mathrm{CH}_{2} \mathrm{Cl}_{2}(4.0 \mathrm{~mL})$ under Ar atmosphere. The reaction mixture was stirred for $15 \mathrm{~min}$ at $-30{ }^{\circ} \mathrm{C}$ before $o$-naphthoquinone $\mathbf{1 b}(0.44 \mathrm{mmol})$ was added under $\mathrm{Ar}$ atmosphere. Then the reaction was stirred vigorously at $-30^{\circ} \mathrm{C}$ until $\mathbf{2 b}$ was completely consumed. Typical reaction time was about $24 \mathrm{~h}$. The resulting mixture was concentrated under reduced pressure and purified by flash chromatography on silica gel (eluent: $\mathrm{PE} / \mathrm{EA}=6 / 1$ ) to give rise to the corresponding axially chiral arylquinones $(R)-3 c$

General procedure for asymmetric synthesis of (S)-3d. To a mixture of $(R)$ CPA7 $(10 \mathrm{~mol} \%)$ and $\mathrm{BF}_{4} \mathrm{Li}(8 \mathrm{~mol} \%)$ in $10 \mathrm{~mL}$ Schlenk tube was added $\mathrm{CH}_{2} \mathrm{Cl}_{2}$ $(4.0 \mathrm{~mL})$ under Ar atmosphere. The reaction mixture was stirred for $1 \mathrm{~h}$ at room temperature. After that, $o$-naphthoquinone $1 \mathrm{a}(0.22 \mathrm{mmol})$ and $2 \mathrm{c}(0.10 \mathrm{mmol})$ were added and the mixture was stirred vigorously at $-10^{\circ} \mathrm{C}$ until $2 \mathrm{c}$ was completely consumed. Typical reaction time was about $24 \mathrm{~h}$. The resulting mixture was concentrated under reduced pressure and purified by flash chromatography on silica gel (eluent: $\mathrm{PE} / \mathrm{EA}=6 / 1$ ) to produce the corresponding axially chiral arylquinones $(S)-3 \mathbf{d}$

General procedure for asymmetric synthesis of (S)-3e. To a mixture of $(R)$ CPA4 (10 mol\%) and indole derivative $2 \mathbf{d}(0.10 \mathrm{mmol})$ in $10 \mathrm{~mL}$ Schlenk tube was added $\mathrm{CH}_{2} \mathrm{Cl}_{2}(1.0 \mathrm{~mL})$ under Ar atmosphere. The reaction mixture was stirred at $0{ }^{\circ} \mathrm{C}$ for $20 \mathrm{~min}$. After that, a solution of $o$-naphthoquinone $1 \mathrm{c}(0.20 \mathrm{mmol})$ in $\mathrm{CH}_{2} \mathrm{Cl}_{2}(1.0 \mathrm{~mL})$ was added dropwise at $0{ }^{\circ} \mathrm{C}$. Then the reaction mixture was stirred vigorously at $0^{\circ} \mathrm{C}$ until $2 \mathbf{d}$ was completely consumed (monitored by thinlayer chromatography). Typical reaction time was about $24 \mathrm{~h}$. The resulting mixture was concentrated under reduced pressure and purified by flash chromatography on silica gel (eluent: $\mathrm{PE} / \mathrm{DCM}=1 / 2$ ) to give the corresponding axially chiral arylquinones $(S)-\mathbf{3 e}$

\section{Data availability}

The X-ray crystallographic coordinates for structures reported in this Article have been deposited at the Cambridge Crystallographic Data Centre (CCDC), under deposition number CCDC 1833698. These data can be obtained free of charge from The Cambridge Crystallographic Data Centre via http://www.ccdc.cam.ac.uk/data_request/ cif. Supplementary information and chemical compound information are available in the online version of the paper. For NMR analysis and HPLC traces of the compounds in this article, see Supplementary Figures. Reprints and permissions information is available online at www.nature.com/reprints. Correspondence and requests for materials should be addressed to B.T

Received: 28 May 2019 Accepted: 27 August 2019

Published online: 19 September 2019

\section{References}

1. Kozlowski, M. C., Morgan, B. J. \& Linton, E. C. Total synthesis of chiral biaryl natural products by asymmetric biaryl coupling. Chem. Soc. Rev. 38, 3193-3207 (2009).
2. Bringmann, G., Gulder, T., Gulder, T. A. M. \& Breuning, M. Atroposelective total synthesis of axially chiral biaryl natural products. Chem. Rev. 111, 563-639 (2011)

3. Clayden, J., Moran, W. J., Edwards, P. J. \& LaPlante, S. R. The challenge of atropisomerism in drug discovery. Angew. Chem. Int. Ed. 48, 6398-6401 (2009).

4. LaPlante, S. R. et al. Assessing atropisomer axial chirality in drug discovery and development. J. Med. Chem. 54, 7005-7022 (2011).

5. $\mathrm{Pu}, \mathrm{L}$. 1,1'-Binaphthyl dimers, oligomers, and polymers: molecular recognition, asymmetric catalysis, and new materials. Chem. Rev. 98, 2405-2494 (1998)

6. $\mathrm{Pu}, \mathrm{L}$. Enantioselective fluorescent sensors: a tale of BINOL. Acc. Chem. Res. 45, 150-163 (2012).

7. Kočovský, P., Vyskočil, Š. \& Smrčina, M. Non-symmetrically substituted 1,1' binaphthyls in enantioselective catalysis. Chem. Rev. 103, 3213--33246 (2003).

8. Ding, K., Li, X., Ji, B., Guo, H. \& Kitamura, M. Ten years of research on NOBIN chemistry. Curr. Org. Syn. 2, 499-545 (2005).

9. Chen, Y., Yekta, S. \& Yudin, A. K. Modified BINOL ligands in asymmetric catalysis. Chem. Rev. 103, 3155-3212 (2003).

10. Brunel, J. M. BINOL: a versatile chiral reagent. Chem. Rev. 105, 857-898 (2005).

11. Noyori, R. \& Takaya, H. BINAP: an efficient chiral element for asymmetric catalysis. Acc. Chem. Res. 23, 345-350 (1990).

12. Akiyama, T. Stronger Brønsted acids. Chem. Rev. 107, 5744-5758 (2007).

13. Terada, M. Binaphthol-derived phosphoric acid as a versatile catalyst for enantioselective carbon-carbon bond forming reactions. Chem. Commun. 35, 4097-4112 (2008).

14. Wencel-Delord, J., Panossian, A., Leroux, F. R. \& Colobert, F. Recent advances and new concepts for the synthesis of axially stereoenriched biaryls. Chem. Soc. Rev. 44, 3418-3430 (2015).

15. Kumarasamy, E., Raghunathan, R., Sibi, M. P. \& Sivaguru, J. Nonbiaryl and heterobiaryl atropisomers: molecular templates with promise for atropselective chemical transformations. Chem. Rev. 115, 11239-11300 (2015).

16. Lv, F. \& Yao, Z.-J. Recent advances in direct dehydrogenative biphenyl couplings. Sci. China Chem. 60, 701-720 (2017).

17. Sairafianpour, M. et al. Leishmanicidal, Antiplasmodial, and cytotoxic activity of novel diterpenoid 1,2-duinones from perovskia abrotanoides: new source of tanshinones. J. Nat. Prod. 64, 1398-1403 (2001).

18. Xu, S. W. \& Liu, P. Q. Tanshinone II-A: new perspectives for old remedies. Expert Opin. Ther. Pat. 23, 149-153 (2013).

19. Mure, M. Tyrosine-derived quinone cofactors. Acc. Chem. Res. 37, 131-139 (2004).

20. Klinman, J. P. \& Boonot, F. Intrigues and intricacies of the biosynthetic pathways for the enzymatic quinocofactors: PQQ, TTQ, CTQ, TPQ, and LTQ. Chem. Rev. 114, 4343-4365 (2014).

21. Largeron, M. Protocols for the catalytic oxidation of primary amines to imines. Eur. J. Org. Chem. 2013, 5225-5235 (2013).

22. Wendlandt, A. E. \& Stahl, S. S. Quinone-catalyzed selective oxidation of organic molecules. Angew. Chem. Int. Ed. 54, 14638-14658 (2015).

23. Hussain, H. H. et al. Development of novel antioxidants: design, synthesis, and reactivity. J. Org. Chem. 68, 7023-7032 (2003).

24. Hung, C. H. et al. Experimental and theoretical studies on iron-promoted oxidative annulation of arylglyoxal with alkyne: unusual addition and migration on the aryl ring. J. Am. Chem. Soc. 139, 17015-17021 (2017).

25. Li, G. Q. et al. Organocatalytic aryl-aryl bond formation: an atroposelective $[3,3]$-rearrangement approach to BINAM derivatives. J. Am. Chem. Soc. 135, 7414-7417 (2013)

26. De, C. K., Pesciaioli, F. \& List, B. Catalytic asymmetric Benzidine rearrangement. Angew. Chem. Int. Ed. 52, 9293-9295 (2013).

27. Barrett, K. T., Metrano, A. J., Rablen, P. R. \& Miller, S. J. Spontaneous transfer of chirality in an atropisomerically enriched two-axis system. Nature 509, 71-75 (2014). 
28. Link, A. \& Sparr, C. Organocatalytic atroposelective aldol condensation: synthesis of axially chiral biaryls by arene formation. Angew. Chem. Int. Ed. 53, 5458-5461 (2014).

29. Hazra, C. K., Dherbassy, Q., Wencel-Delord, J. \& Colobert, F. Synthesis of axially chiral biaryls through sulfoxide-directed asymmetric mild $\mathrm{C}-\mathrm{H}$ activation and dynamic kinetic resolution. Angew. Chem. Int. Ed. 53, 13871-13875 (2014).

30. Feng, J., Li, B., He, Y. \& Gu, Z.-H. Enantioselective synthesis of atropisomeric vinyl arene compounds by palladium catalysis: a carbene strategy. Angew. Chem. Int. Ed. 55, 2186-2190 (2016).

31. Yu, C.-G., Huang, H., Li, X.-M., Zhang, Y.-T. \& Wang, W. Dynamic kinetic resolution of biaryl lactones via a chiral bifunctional amine thiourea-catalyzed highly atropo-enantioselective transesterification. J. Am. Chem. Soc. 138, 6956-6959 (2016).

32. Mori, K., Itakura, T. \& Akiyama, T. Enantiodivergent atroposelective synthesis of chiral biaryls by asymmetric transfer hydrogenation: chiral phosphoric acid catalyzed dynamic kinetic resolution. Angew. Chem. Int. Ed. 55, 11642-11646 (2016).

33. Wang, J., Chen, M.-W., Ji, Y., Hu, S.-B. \& Zhou, Y.-G. Kinetic resolution of axially chiral 5- or 8-substituted quinolines via asymmetric transfer hydrogenation. J. Am. Chem. Soc. 138, 10413-10416 (2016).

34. Olliffe, J. D., Armstrong, R. J. \& Smith, M. D. Catalytic enantioselective synthesis of atropisomeric biaryls by a cation-directed O-alkylation. Nat. Chem. 9, 558-562 (2017).

35. Wang, Y.-B. \& Tan, B. Construction of axially chiral compounds via asymmetric organocatalysis. Acc. Chem. Res. 51, 534-547 (2018).

36. Akiyama, T., Itoh, J., Yokota, K. \& Fuchibe, K. Enantioselective Mannich-type reaction catalyzed by a chiral Brønsted acid. Angew. Chem. Int. Ed. 43, 1566-1568 (2004).

37. Uraguchi, D. \& Terada, M. Chiral Brønsted acid-catalyzed direct Mannich reactions via electrophilic activation. J. Am. Chem. Soc. 126, 5356-5357 (2004).

38. Chen, Y.-H. et al. Atroposelective synthesis of axially chiral biaryldiols via organocatalytic arylation of 2-naphthols. J. Am. Chem. Soc. 137, 15062-15065 (2015).

39. Goriya., Y., Kim, H.-Y. \& Oh, K. o-Naphthoquinone-catalyzed aerobic oxidation of amines to (ket)imines: a modular catalyst approach. Org. Lett. 18, 5174-5177 (2016).

40. Moliterno, M. et al. Quinine-catalyzed asymmetric synthesis of 2,2'binaphthol-type biaryls under mild reaction conditions. Angew. Chem. Int. Ed. 55, 6525-6529 (2016).

41. Wang, J.-Z. et al. Symmetry in cascade chirality-transfer processes: a catalytic atroposelective direct arylation approach to BINOL derivatives. J. Am. Chem. Soc. 138, 5202-5205 (2016).

42. You, S.-L., Cai, Q. \& Zeng, M. Chiral Brønsted acid catalyzed Friedel-Crafts alkylation reactions. Chem. Soc. Rev. 38, 2190-2201 (2009).

43. Bartoli, G., Bencivenni, G. \& Dalpozzo, R. Organocatalytic strategies for the asymmetric functionalization of indoles. Chem. Soc. Rev. 39, 4449-4465 (2010).

44. Harris, G. D. et al. A one-pot, two-step synthesis of tetrahydro Asterriquinone E. Org. Lett. 1, 431-434 (1999).

45. Pirrung, M. C., Park, K. \& Li, Z.-T. Synthesis of 3-indolyl-2,5dihydroxybenzoquinones. Org. Lett. 3, 365-367 (2001).

46. Laursen, J. B. \& Nielsen, J. Phenazine natural products: biosynthesis, synthetic analogues, and biological activity. Chem. Rev. 104, 1663-1686 (2004).

47. Beifuss, U. \& Tietze, M. Methanophenazine and other natural biologically active phenazines. Top. Curr. Chem. 244, 77-113 (2005).
48. Guttenberger, N., Blankenfeldt, W. \& Breinbauer, R. Recent developments in the isolation, biological function, biosynthesis, and synthesis of phenazine natural products. Bioorg. Med. Chem. 25, 6149-6166 (2017).

49. Quinonero, O. et al. Combining organocatalysis with central-to-axial chirality conversion: atroposelective Hantzsch-type synthesis of 4-arylpyridines. Angew. Chem. Int. Ed. 55, 1401-1405 (2016).

50. Raut, V. S. et al. Enantioselective syntheses of furan atropisomers by an oxidative central-to-axial chirality conversion strategy. J. Am. Chem. Soc. 139, 2140-2143 (2017)

\section{Acknowledgements}

Financial support from the National Natural Science Foundation of China (Nos. 21772081 and 21825105), Shenzhen Nobel Prize Scientists Laboratory Project (C17213101), Shenzhen Special Funds for the Development of Biomedicine, Internet, New Energy, and New Material Industries (JCYJ20170412151701379,

KQJSCX20170328153203) is greatly appreciated.

\section{Author contributions}

S.Z., Y.-H.C., and Y.-B.W. designed and performed experiments and prepared the supplementary information. S.-H.X. and S.-Y.L. helped with characterizing some new compounds. B.T., J.-Q.W., and J.X. conceived and directed the project and S.-H.X., P.Y., and B.T. wrote the paper. All authors discussed the results and commented on the manuscript.

\section{Additional information}

Supplementary Information accompanies this paper at https://doi.org/10.1038/s41467019-12269-4.

Competing interests: The authors declare no competing interests.

Reprints and permission information is available online at http://npg.nature.com/ reprintsandpermissions/

Peer review information Nature Communications thanks the anonymous reviewers for their contribution to the peer review of this work.

Publisher's note Springer Nature remains neutral with regard to jurisdictional claims in published maps and institutional affiliations.

Open Access This article is licensed under a Creative Commons Attribution 4.0 International License, which permits use, sharing, adaptation, distribution and reproduction in any medium or format, as long as you give appropriate credit to the original author(s) and the source, provide a link to the Creative Commons license, and indicate if changes were made. The images or other third party material in this article are included in the article's Creative Commons license, unless indicated otherwise in a credit line to the material. If material is not included in the article's Creative Commons license and your intended use is not permitted by statutory regulation or exceeds the permitted use, you will need to obtain permission directly from the copyright holder. To view a copy of this license, visit http://creativecommons.org/ licenses/by/4.0/

(C) The Author(s) 2019 